\title{
\begin{tabular}{ll} 
Research Square & Preprints are preliminary reports that have not undergone peer review. \\
\hline & They should not be considered conclusive, used to inform clinical practice,
\end{tabular} or referenced by the media as validated information.
}

\section{Optimizing the radiation treatment planning of brain tumors by integration of Functional MRI and White Matter Tractography}

\section{Arman Boroun}

Radiation Sciences Research Center (RSRC), Aja University of Medical Sciences, Tehran

Alireza Montazerabadi

Medical Physics Research Center, Mashhad University of Medical Sciences, Mashhad

Seyed Hadi Molana

Department of Radiation Oncology, Aja University of Medical Sciences, Tehran

Hamid Gholamhosseinian

Medical Physics Research Center, Mashhad University of Medical Sciences, Mashhad

Fakhereh Pashaei ( $\nabla$ fakhereh.pashaee@gmail.com )

Radiation Sciences Research Center (RSRC), Aja University of Medical Sciences, Tehran

\section{Research Article}

Keywords: Brain Tumor, fMRI, Diffusion Tractography, Radiation Therapy

Posted Date: June 21st, 2022

DOI: https://doi.org/10.21203/rs.3.rs-1373965/v3

License: (c) (1) This work is licensed under a Creative Commons Attribution 4.0 International License. Read Full License 


\section{Abstract}

Purpose: The purpose of this study was to assess whether the incorporation of functional magnetic resonance imaging (fMRI) and diffusion tensor imaging (DTI) data into the radiation treatment planning process of brain tumors could spare the neurological parts of the brain from high doses of radiation.

Methods: In this study, the fMRI and DTI data were obtained from eight glioma patients and included in the radiation treatment plan. This patient-specific fMRI and DTI data were attained based on tumor location, patient's general conditions, and the importance of the functional and fiber tract areas. The functional regions, fiber tracts, anatomical organs at risk, and the tumor were contoured for radiation treatment planning. Finally, the radiation treatment planning with and without fMRI \& DTI information was obtained and compared.

Results: On average, the mean dose to the functional areas was reduced by $25.36 \%$, and the maximum dose was reduced by $18.57 \%$ on $\mathrm{fMRI} \&$ DTI plans compared with the anatomical plans In addition, $15.59 \%$ and $20.84 \%$ reductions in the mean and maximum doses of the fiber tracts were achieved, respectively.

Conclusion: This study demonstrated the feasibility of using fMRI and DTI data in radiation treatment planning to maximize radiation protection of the functional cortex and fiber tracts. We also observed a significant reduction in the mean and maximum doses to these neurologically relevant brain regions. Dose reduction would reduce the neuro-cognitive complications and improve the patient's quality of life.

\section{Introduction}

Gliomas are the largest group of primary intracranial tumors (1). Low-grade gliomas (LGGs) are typically slow-growing primary brain tumors, but they have a very heterogeneous clinical behavior (2). Compared with low-grade gliomas, high-grade gliomas have a poor prognosis, a higher degree of malignancy, and are more difficult to control (3). Gliomas make up one-third of all primary tumors. However, they represent $80 \%$ of all malignant primary brain tumors, which receive several therapeutic strategies, including surgery, radiotherapy, chemotherapy, or combinations (3).

Three-dimensional conformal radiation therapy (3DCRT) has been recognized as the standard therapy for glioma patients, including high-dose megavoltage-range radiation to the tumor $(4,5)$. Approximately half of the patients survive $>6$ months, and many patients achieve long-term control or cure (5). Cranial radiation can cause acute and chronic damage to various cells, resulting in the demyelination and necrosis of the brain regions (6). Neurocognitive dysfunction is one of the main side effects of cranial irradiation that seriously affects cognitive function and quality of life (QOL) $(7,8)$. In $50-90 \%$ of patients receiving fractional radiotherapy, cognitive dysfunctions related to memory, executive functions, sustained attention, and information processing speed has been observed $(5,9)$. 
Recent advances in multimodality treatment protocols have significantly improved the survival rates of patients with brain tumors (10). Despite these advances in treatment approaches, CRT directly damages white and grey matter by causing inflammation, angiogenesis, and cell death (11). The identification, characterization, and minimization of specific side effects, especially those related to functional sequelae, can seriously affect the quality of life (12).

The prevention or minimization of radiotherapy-induced cognitive dysfunction has been a topic of intense research for a long time (13). Some successful strategies to reduce neurocognitive dysfunction side effects include avoiding radiotherapy in young children and reducing radiation dose and brain volume exposure (14).

Modern radiation treatment plans are based on a conventional MRI scan co-registration with a planning CT scan. Integrating functional imaging and white matter tractography for fractionated radiotherapy planning in brain tumors is progressing slowly (13). When radiotherapy techniques combine with advanced imaging such as $\mathrm{fMRI}$ and DTI, they can bypass potential brain areas involved in neurocognitive functions (13). With the aid of modern magnetic resonance imaging (MRI) technology, the functional areas of the brain and white matter fiber pathways can be well imaged (15). Blood oxygen level-dependent (BOLD) functional MRI (fMRI) and white matter tractography have been used to identify functional structures and white-matter pathways of the brain, respectively, as critical volumes in treatment planning $(16,17)$. Integrating these modern techniques into radiation treatment plans could help spare healthy and sensitive brain areas from high-dose radiation (16-18). When the tumor is located in critical regions, nearby functional structures and white matter pathways can receive radiation doses beyond their tolerance limits because they cannot be easily identified on anatomical CT/ MRI images. Functional neuroimaging, including blood oxygen level-dependent (BOLD) and white matter tractography, can identify functional structures and white matter pathways; these imaging techniques are implemented in the radiation treatment plans to prevent the beam from passing through nearby critical areas, thereby reducing radiation doses (19).

This investigation aimed to selectively protect the functional and neural pathways in patients with primary brain tumors by integrating functional MRI (fMRI) and white matter tractography into the threedimensional conformal radiotherapy (3DCRT). Radiation dose distributions with and without functional structures and white matter fiber pathways developed and compared.

\section{Materials And Methods}

\subsection{Patients}

According to pre-operative MRI scans, a total of 8 suspected glioma patients (five male patients and three female patients aged from 21 to 58), who are scheduled for tumor resection and provide their informed consent, were recruited from December 2019 to June 2020. The glioma tissues in our study included four low-grade gliomas (WHO Grade II) and four high-grade gliomas (WHO Grade IV). The patients' characteristics are shown in Table 1. 
Table 1

The Characteristics of the Patients

\begin{tabular}{|llllll|}
\hline Patient & Gender & Age of diagnosis (y) & Lesion location a $^{2}$ & Tumor type & Grade \\
\hline 1 & Male & 29 & F-T & Glioma & High \\
2 & Female & 24 & F & Glioma & Low \\
3 & Male & 58 & T & Glioma & Low \\
\hline 4 & Female & 26 & P & Glioma & Low \\
\hline 5 & Female & 18 & F-P & Glioma & High \\
6 & Female & 54 & P & Glioma & Low \\
\hline 7 & Female & 63 & F & Glioma & High \\
8 & Female & 34 & F-P & Glioma & High \\
\hline
\end{tabular}

a Note: F, frontal; T, temporal; P, parietal; O, occipital

All patients underwent pre-radiotherapy Functional MRI as well as DTI and conventional MRI. Functional structures and white-matter pathways were placed in the natural regions without any overlap with the target volume of the lesions. All patients were able to perform the specific tasks to generate functional maps of brain activation. The Varian linear accelerator with a Millennium dynamic multileaf collimator (DMLC) system delivered external radiation therapy.

\subsection{Patient preparation and MRI test considerations}

The MR scanning sequence was begun by screening the patient for any clothing, jewelry, or devices such as pacemakers that may degrade the MR images and MRI safety and patient's condition evaluation. The technologist explained the scanning procedure and answered questions. Patients were advised not to move at all during the examination. Also, those heads were kept in a fixed position with special pads as much as possible. To order the paradigms, we tried to prioritize the difficulty and simplicity of implementation. Achieving the desired outcomes requires focusing on a paradigm needing more patient's attention at first, then simpler paradigms such as finger movement implementation.

\subsection{Data acquisition}

Three imaging studies were performed on the patients using a Siemens MAGNETOM Avanto 1.5 T scanner with an eight-channel head coil.

\subsubsection{Anatomical and Functional MRI characteristics}


T1-weighted structural brain MR Images were initially obtained using the MP-RAGE sequence. BOLD fMRI data were acquired to provide brain activation maps, using single-shot gradient echo-planar imaging (EPI), which is more sensitive to $\mathrm{T} 2{ }^{*}$ changes. The structural and functional MRIs were performed using the parameters summarized in Table 2.

\section{Table 2.}

The characteristics of the structural images and functional MRI

\begin{tabular}{|llllllll|}
\hline Sequence & $\begin{array}{l}\text { TR } \\
(\mathbf{m s})\end{array}$ & $\begin{array}{l}\text { TE } \\
(\mathbf{m s})\end{array}$ & $\begin{array}{l}\text { TI } \\
(\mathbf{m s})\end{array}$ & $\begin{array}{l}\text { Slice } \\
\text { Thickness }(\mathrm{mm})\end{array}$ & $\begin{array}{l}\text { Number of } \\
\text { Slices }\end{array}$ & FA & Matrix \\
\hline MP-RAGE & 1820 & 3.49 & 1100 & 1 & 176 & $7^{\circ}$ & $256 \star 256$ \\
\hline GRE-EPI & 3000 & 30 & - & 3 & 176 & $90^{\circ}$ & $65 \star 64$ \\
\hline
\end{tabular}

\subsubsection{Diffusion Tensor Imaging (DT)}

DTI images acquired with the spin echo-echo planar imaging (SE-EPI) sequence (TR $=9500 \mathrm{~ms}, \mathrm{TE}=90$ $\mathrm{ms}$, acquisition matrix $=128 \times 128$ pixels; FOV $=256 \mathrm{~mm} \times 256 \mathrm{~mm}^{2}$; slice thickness $=2.0 \mathrm{~mm}$; $\mathrm{b}$ value of $1,000 \mathrm{~s} / \mathrm{mm}^{2}$ along 12 non-collinear directions).

\subsubsection{Experimental Paradigms}

This study simulated the primary motor cortex, Broca's area, and Wernicke's area. The motor cortex, located in the precentral gyrus, is mainly responsible for the actual performance of movements (20). Broca's area, which is placed in the frontal lobe (often left), produces speech. In contrast, Wernicke's area in the temporal lobe (usually the left side) is related to language comprehension and speech planning (21).

The hand, foot, lip, and tongue motor paradigms activated the primary motor cortex. The measurement sequence of these paradigms consisted of a 24-second activation period (8 scans) followed by a 24second rest period (8 scans), with eight times of repeat. All the measurements started with an initial rest period.

The reverse word reading (RWR) paradigm was used to stimulate Broca's area. The stimuli of the RWR task in each activation block consisted of 10-word trials in 24 seconds, followed by a 24-seconds rest period. The subject is presented with a five-letter Persian word in each activation period while the letters were displayed in reverse order. They were then asked to read each word silently once. The measurements always began with an initial rest period (22). The story paradigm was used to stimulate Wernicke's area. Like other designs, this paradigm started with a rest period. In the story paradigm, a short and manageable story was first considered, which was broadcast through the device's audio system during a 
33 second activation period (11 scans) followed by a 33 second rest period (11 scans), and these periods were repeated five times. The story was chosen so that the story would end with the end of the paradigm.

\subsection{Data Analysis}

\subsection{1 fMRI Data Analysis}

After imaging, data were transferred to the MATLAB workstation for analysis using SPM12 software (Statistical Parametrical Mapping, Wellcome Department of Cognitive Neurology, London, England). Data series were motion-corrected and smoothed with a $6 \mathrm{~mm}$ FWHM (full width at half maximum) Gaussian kernel. The model function was applied to each voxel in the brain (general linear model), and finally, a statistical map was estimated. Significantly activated voxels were identified using an initial $p$-value threshold of 0.001. fMRI scan data (mean image) was combined with the T1 structural MRI image obtained at the same scanning position, allowing the functional MR activation color map visibility on the patient's brain anatomy. The merged image sets (JPEG format) were then converted into DICOM format using MATLAB software. In this conversion, the color images were replaced with the grayscale images using the intensity of the green color as the grayscale intensity. fMRI DICOM series were also sent to the planning system.

\subsubsection{DTI Analysis}

Data pre-processing consists of raw data conversion, skull-stripping, and motion/eddy correction was performed using DSI studio software (developed by Fang-Cheng Yeh from the Advanced Biomedical MRI Lab, National Taiwan University Hospital, Taiwan, Supported by Fiber Tractography Lab, University of Pittsburgh, and made available at http://dsistudio. labsolver.org/Download/). After data pre-processing, we used a reconstruction model base (DTI) to process the diffusion images. Average DTI values were extracted from voxels within the ROls and tracts. The tractography process used a deterministic fiber tracking algorithm. Desired neural pathways were extracted in two directions in two hemispheres of the brain. Finally, by using 3D Slicer software, the location of fiber tracts saved as the region of interest (ROI) was determined on the T1 structural images.

\subsection{Image Fusion}

As shown in figure 1. each patient's axial CT images were registered with the corresponding anatomical MRI images, using the automatic registration algorithm of the TPS to precisely describe the targets and standard organs at risk (OARs). The anatomical MRI volumes were fused with the corresponding white matter tracts and fMRI activation maps and then imported into the Isogray treatment planning system (TPS) software version 3.1 as separate grayscale DICOM images.

\subsection{Treatment Planning}

The prescribed dose was 54 Gy to 60 Gy. Anatomical OARs including the brainstem, spinal cord, eyes, bilateral lens, bilateral retina, lacrimal glands, bilateral cochlea, bilateral optic nerves, and the optic 
chiasm were delineated. The fiber tracts and the functional structures nearby the targets were also contoured as extra OARs using the fused $\mathrm{fMRI}$ and DTI images. Two three-dimensional conformal radiotherapy (3DCRT) treatment plans were developed for each patient. In the first plan, only the PTV and anatomical OARs were considered. On the other plan, the physicist took the functional structures and fiber tracts situated near the target as Neurocognitive functions OARs. The second plan was generated to reduce the dose of the functional structures and fiber tracts while keeping prescription dose coverage for the planning target volume (PTV) and similar OAR dose-volume levels as the standard care treatment plan.

Dose-volume histograms (DVH) data were extracted from TPS. The mean ( $\left.D_{\text {mean }}\right)$ and maximum doses $\left(D_{\max }\right)$ of radiation to the functional regions and fiber tracts were determined for both RT plans.

Anatomical OARs and Neurocognitive functions OARs were compared based on the values of $D_{\max }$ and $D_{\text {mean }}$.

\subsection{Statistical analyses}

Wilcoxon's test was used to compare two plans parameters. The differences were considered statistically significant at $p<0.05$. Statistical analyses were performed using SPSS software ver. 25.0.

\section{Results}

In this study, the functional regions and fiber tracts that overlap with the target volume were eliminated due to the priority of tumor treatment. The prescribed dose and the dose that covered 95\% (D95) of the PTV are shown in Table 3. PTV received more than $95 \%$ of the prescribed dose for all patients, both for the original and optimized plan (Figure 2).

\section{Table 3}

The prescription dose and D95 of the PTV 


\begin{tabular}{|lllll|}
\hline $\begin{array}{l}\text { Prescription } \\
\text { Dose (Gy) }\end{array}$ & $\begin{array}{l}\text { D95 of the PTV in plan without } \\
\text { fMRI \& DTI (Gy) }\end{array}$ & $\begin{array}{l}\text { D95 of the PTV in plan with } \\
\text { fMRI \& DTI (Gy) }\end{array}$ & $\begin{array}{l}\text { P- } \\
\text { value }\end{array}$ \\
\hline $\begin{array}{l}\text { Patient } \\
2\end{array}$ & 54 & 57.81 & 57.62 & 0.293 \\
\hline $\begin{array}{l}\text { Patient } \\
3\end{array}$ & 54 & 52.15 & 51.87 \\
\hline $\begin{array}{l}\text { Patient } \\
4\end{array}$ & 54 & 52.18 & 52.41 \\
\hline $\begin{array}{l}\text { Patient } \\
5\end{array}$ & 60 & 52.38 & 51.36 \\
\hline $\begin{array}{l}\text { Patient } \\
6\end{array}$ & 54 & 58.09 & 58.37 \\
\hline $\begin{array}{l}\text { Patient } \\
7\end{array}$ & 60 & 52.36 & 52.72 \\
\hline $\begin{array}{l}\text { Patient } \\
8\end{array}$ & 60 & 58.64 & 57.51 \\
\hline SD & 3.21 & 59.45 & 58.18 \\
\hline Median & 57 & 3.36 & 3.15 \\
\hline
\end{tabular}

The maximum and the mean dose to the anatomical OARs are shown in table 4 and table 5 . The doses received by the anatomical OARs did not exceed the permissible values, both for the optimized and original plans.

Table 4

Maximum Dose to anatomical OARs 


\begin{tabular}{|lll|}
\hline Anatomical OARs & Maximum Dose in original plan (Gy) & Maximum Dose in optimized plan (Gy) \\
\hline Brain Stem & 32.68 & 30.80 \\
\hline Optic Chiasm & 26.17 & 22.44 \\
\hline Left Optic Nerve & 9.84 & 12.77 \\
\hline Right Optic Nerve & 4.25 & 3.08 \\
\hline Left cochlea & 2.34 & 2.96 \\
\hline Right cochlea & 1.70 & 1.12 \\
\hline Left eye & 5.34 & 8.97 \\
\hline Right eye & 2.13 & 2.60 \\
\hline
\end{tabular}

\section{Table 5}

Mean Dose to anatomical OARs

\begin{tabular}{|lll|}
\hline Anatomical OARs & Mean Dose in original plan (Gy) & Mean Dose in optimized plan (Gy) \\
\hline Brain stem & 5.04 & 3.68 \\
\hline Optic chiasm & 13.74 & 11.29 \\
\hline Left optic nerve & 5.43 & 6.01 \\
\hline Right optic nerve & 2.83 & 2.59 \\
\hline Left cochlea & 1.97 & 2.44 \\
\hline Right cochlea & 1.48 & 0.99 \\
\hline Left eye & 2.30 & 3.52 \\
\hline Right eye & 1.73 & 2.14 \\
\hline
\end{tabular}

The details of mean and maximum dose reduction to each patient's functional structures and fiber tracts are presented in table 6 and table 7 . The dose-volume histogram of patient number 8 demonstrates the feasibility of dose reduction in the right optic radiation and left-hand motor without the PTV dose coverage changing (Figure 3).

\section{Table 6}

$D_{\text {mean }}$ and $D_{\max }$ to the Functional Activation Areas 


\begin{tabular}{|c|c|c|c|c|}
\hline $\begin{array}{l}\text { Functional } \\
\text { areas }\end{array}$ & $\begin{array}{l}D_{\text {mean }} \text { in original } \\
\text { plan (Gy) }\end{array}$ & $\begin{array}{l}D_{\text {mean }} \text { in optimized } \\
\text { plan (Gy) }\end{array}$ & $\begin{array}{l}D_{\max } \text { in original } \\
\text { plan (Gy) }\end{array}$ & $\begin{array}{l}D_{\max } \text { in optimized } \\
\text { plan (Gy) }\end{array}$ \\
\hline $\begin{array}{l}\text { Right Hand } \\
\text { Motor }\end{array}$ & 23.94 & 18.99 & 30.29 & 24.76 \\
\hline $\begin{array}{l}\text { Left Hand } \\
\text { Motor }\end{array}$ & 30.65 & 21.78 & 38.44 & 28.94 \\
\hline $\begin{array}{l}\text { Right Lip } \\
\text { Motor }\end{array}$ & 31.33 & 24.20 & 33.29 & 28.29 \\
\hline $\begin{array}{l}\text { Left Lip } \\
\text { Motor }\end{array}$ & 25.35 & 16.87 & 33.52 & 26.58 \\
\hline $\begin{array}{l}\text { Right Tongue } \\
\text { Motor }\end{array}$ & 23.30 & 18.59 & 26.82 & 21.71 \\
\hline $\begin{array}{l}\text { Left Tongue } \\
\text { Motor }\end{array}$ & 20.43 & 13.18 & 26.46 & 21.91 \\
\hline Right Broca & 55.38 & 50.77 & 57.06 & 56.52 \\
\hline Left Broca & 11.06 & 5.51 & 14.98 & 6.54 \\
\hline $\begin{array}{l}\text { Right } \\
\text { Wernicke }\end{array}$ & 23.36 & 16.91 & 34.18 & 28.04 \\
\hline Left Wernicke & 8.67 & 2.38 & 7.98 & 3.46 \\
\hline Mean & 25.35 & 18.92 & 30.30 & 24.68 \\
\hline SD & 12.83 & 13.10 & 13.23 & 14.36 \\
\hline Median & 23.65 & 17.75 & 31.79 & 25.67 \\
\hline
\end{tabular}

Table 7

Dmean and Dmax to the Fiber Tracts 


\begin{tabular}{|lllll|}
\hline Fiber Tracts & $\begin{array}{l}\mathrm{D}_{\text {mean }} \text { in } \\
\text { original plan } \\
(\mathrm{Gy})\end{array}$ & $\begin{array}{l}\mathrm{D}_{\text {mean }} \text { in } \\
\text { optimized plan } \\
(\mathrm{Gy})\end{array}$ & $\begin{array}{l}\mathrm{D}_{\text {max }} \text { in } \\
\text { original plan } \\
(\mathrm{Gy})\end{array}$ & $\begin{array}{l}\mathrm{D}_{\text {max }} \text { in } \\
\text { optimized plan } \\
(\mathrm{Gy})\end{array}$ \\
\hline Right Corticospinal Tract & 10.24 & 5.32 & 15.07 & 8.62 \\
\hline Left Corticospinal Tract & 36.14 & 32.89 & 55.54 & 55.59 \\
\hline Right Frontal Aslant Tract & 14.24 & 8.87 & 36.57 & 27.12 \\
\hline Left Frontal Aslant Tract & 27.42 & 24.84 & 47.55 & 43.90 \\
\hline $\begin{array}{l}\text { Right Geniculocalcarine } \\
\text { tract (Optic Radiation) }\end{array}$ & 14.69 & 8.64 & 30.81 & 25.83 \\
\hline $\begin{array}{l}\text { Left Geniculocalcarine } \\
\text { tract (Optic Radiation) }\end{array}$ & 15.09 & 12.95 & 49.74 & 46.03 \\
\hline Right Uncinate Fasciculus & 13.02 & 10.06 & 53.52 & 36.70 \\
\hline Mean & 18.69 & 14.80 & 41.26 & 34.83 \\
\hline SD & 9.43 & 10.14 & 14.62 & 15.65 \\
\hline Median & 14.69 & 10.06 & 47.55 & 36.70 \\
\hline
\end{tabular}

The results indicated that the average reduction of the mean and maximum doses to the optimized plans' functional areas was $25.36 \%(P=0.005)$ and $18 \%(P=0.005)$, respectively, compared with the original plans. In addition, compared to the original plans, the average reduction of the mean and maximum doses to the optimized plans' fiber tracts was $15.59 \%(P=0.028)$ and $20.84 \%(P=0.018)$, respectively. In summary, the results showed significant dose reduction in the functional structure and fiber tracts while radiotherapy planning target volume (PTV) maintained the prescribed dose. Moreover, the anatomical OARs were kept strictly within the acceptable dose tolerance.

\section{Discussion}

While radiation therapy has dramatically improved local control and prolonged progression-free patient survival in several cancer types including, primary brain tumor, radiation-induced cognitive impairment is considered a late effect of radiation therapy (RT) in most brain tumor patients because of neuro-cognitive areas' high doses receiving $(1,2,3)$. Neurocognitive function damage is one of cranial irradiation's most crucial side effects that play an essential role in cognitive function and quality of life $(\mathrm{QOL})(7,8)$. Affected cognitive domains involve IQ scores, learning, memory, processing speed, attention, and executive functioning with a consequent increasing need for rehabilitation interventions (4). Furthermore, neuropsychological effects may include social, emotional, and behavioral disorders (5), leading to a significantly decreased quality of life than peers (6). Radiation-induced brain injury, including 
inflammation, angiogenesis, and cell death, can result in white and grey matter dysfunctions (7) and RT brain damage, causing demyelination (or structural degradation) of axon fibers and disruption of transsynaptic communications (8). These injuries may lead to severe irreversible neurological consequences without any visible sign on conventional neuroimaging and histopathology (9), and they harm the quality of life and daily functioning (10). Currently, knowledge about cognitive dysfunction following cranial radiation is limited (11); However, $19-83 \%$ of fractionated radiotherapy survivors exhibit disabling and progressive cognitive dysfunction (1). According to the advanced imaging technique, a clinically feasible protocol has been introduced to improve the extent of resection in glioma surgery involving the cortical motor areas and subcortical white matter tracts to improve patients' quality of life. The benefit of Modern brain tumor surgery was more noticeable for neurosurgeons with less experience to identify and ultimately preserve vital sites while standing in the pillar of maximum safe resection. Neurosurgery has tried to avoid neuro dysfunction during surgery, but there is no clear strategy for radiotherapy. Because of a late manifest of radiation-induced neurocognitive injury and the short-term survival of glioma patients, there has not been noticed as it should be to use new imaging advances in radiotherapy treatment planning. While many glioma patients die before the onset of neurological complications, the latest advancements in treatment techniques and widespread advances in neuroimaging; lead to an increase in life expectancy of glioma patients. Integration of DTI and BOLD-fMRI have been used to identify white matter pathways and functional brain structures recently. In this study, we demonstrated that considering functional structures and fiber tracts during treatment planning could clinically reduce the radiation doses and neurocognitive complications and increase the patient's life expectancy quality of life. Liu et al. have reported an innovative method to combine the fMRI brain activation map for stereotactic radiosurgery (SRS) plans. In the SRS plan, direct exposure to the eloquent cortex through multiple radiation arcs or static radiation IMRT beams was avoided, and the average dose of the eloquent cortex was reduced to $32 \%$ (13). Pantelis et al. has demonstrated that with the help of the integration of BOLD-fMRI and DTI into CyberKnife stereotactic radiosurgery can be considered and spared critical brain structures (14). Wang et al. integrated BOLD-fMRI and DTI data in radiation treatment plans of 20 patients with high-grade glioma. fMRI and DTI data were derived from the primary motor cortex and the corticospinal tract, respectively.

They have found that the Dmax and Dmean of the ipsilateral and contralateral PMC and CST regions were considerably decreased (15). fMRI and DTI data of 16 patients with brain tumors applied in CyberKnife radiosurgery by Sun et al. fMRI data were obtained from the speech area and DTI data from the pyramidal tracts. It was observed that the maximum dose in the functional area was reduced by $22.71 \%$ on average $(16)$.

Rhodes et al. were used resting-State fMRI data from 9 patients with primary brain tumors in their radiation therapy plans. Functional data in the default mode network (DMN) was obtained from restingstate $\mathrm{fMRI}$. On average, they achieved $20 \%(P=0.002)$ and $12 \%(P=0.002)$ reductions in the mean and maximum doses, respectively, to the DMN (17).

In most similar studies, the radiation therapy design considered the same functional area and a neural tract for all patients. Our study treatment plans found several functional areas and neural tract according 
to each patient's tumor site and functional performance. When the tumor's location was noticed to choosing functional areas and neural tracts, a significant dose reduction was observed in the optimized plan; also, useless information was omitted.

The integration of functional MRI and diffusion tensor imaging in radiation treatment planning have many challenges and limitations that need to be considered. These challenges are more complex for patients with brain tumors.

A patient's participation in performing functional tasks for imaging data acquisition, high cost advanced imaging, and time-consuming and complicated treatment plans because of adding functional organs at risk and neural tracts are challenges. Large tumor volume may limit our radiation treatment plan. It will be challenging to fully encompassed PTV by $95 \%$ of the prescribed dose while minimizing the dose delivered to the specified organs at risk. As a result, it cannot be possible to consider high-risk functional areas and neural tracts in radiation treatment plans.

Patient survival should also be considered. Since the neurocognitive complications are late radiation effects, they are at the lowest risk for short-term survival. It would be useless to involve the patient with advanced image acquisition, which adds additional cost and complexity in this condition. Therefore, patients with adequate General health conditions, local tumors, and relatively long survival are the best cases for advanced treatment planning with fMRI and DTI. The future of cancer radiotherapy will depend on the usage of advanced imaging in modern treatment planning techniques. The more precise combination, the more beneficial and effective treatment will be. This study results can provide the basis for future clinical follow-up studies to more precisely investigate the impacts of dose reduction on neurological-cognitive complications.

\section{Conclusion}

As the finishing statement, it has to be mentioned that the functional regions and fiber tracts can be integrated into the radiation treatment planning for patients with brain tumors. This integration leads to a notable dose reduction to the critical areas, thereby preserving the unique function of the brain without compromising the PTV coverage or sparing of anatomical organs at risk. For the clinical use of this method, there is no need to combine the functional regions and fiber tracts in radiation treatment planning for all patients with brain tumors. Several factors prioritize patients, such as tumor type, grade, and location, together with the patient's age, survival, and physical status.

Altogether, a proprietary integration of $\mathrm{fMRI}$ and DTI into radiation treatment planning is possible, necessary, and valuable. Determining this necessity is the responsibility of a team of neurologists, radiologists, medical physicists, and radiation oncologists.

\section{Declarations}

\section{Acknowledgments:}


This research was supported by Radiation Sciences Research Center (RSRC), Aja University of Medical Sciences, (Tehran, Iran). It was approved by the Ethics Committee of Aja University of Medical Sciences, with the registration number of "IR.AJAUMS.REC.1399.255".

\section{Competing interests' statement}

We declare that this manuscript is original, has not been published before, and is not currently being considered for publication elsewhere. We wish to confirm that there are no known conflicts of interest associated with this publication. We confirm that the manuscript has been read and approved by all named authors and that there are no other persons who satisfied the criteria for authorship but are not listed. The authors alone are responsible for the content and writing of the paper. We further confirm that the order of authors listed in the manuscript has been approved by all of the authors.

\section{Funding declaration}

There has been no significant financial support for this work that could have influenced its outcome.

\section{Author Contribution}

All authors contributed to the study's conception, methodology, and design. Material preparation and data collection were performed by ARMAN BOROUN and Hamid Gholamhosseinian. Data analysis were performed by ARMAN BOROUN and Alireza Montazerabadi. The main manuscript text was written by ARMAN BOROUN and Fakhereh Pashaei. Alireza Montazerabadi (medical physicist- MRI) and Seyed Hadi Molana (Radiation oncologist) and Hamid Gholamhosseinian (medical physicist- radiation therapy) reviewed and edited the paper according to their field. All authors read and approved the final manuscript.

\section{References}

1. Sontheimer H. Chapter 9 - Brain Tumors. In: Sontheimer H editor. Diseases of the Nervous System. San Diego: Academic Press; 2015. p. 259-88.

2. Pignatti F, Van Den Bent M, Curran D, Debruyne C, Sylvester R, Therasse P, Afra D, Cornu P, Bolla M, Vecht C, Karim AB. Prognostic factors for survival in adult patients with cerebral low-grade glioma. Journal of Clinical Oncology. 2002 Apr 15;20(8):2076-84.

3. Chang J, Narayana A. Functional MRI for radiotherapy of gliomas. Technol Cancer Res Treat. 2010;9(4):347-58.

4. Wang M, Ma H, Wang X, Guo Y, Xia X, Xia H, Guo Y, Huang X, He H, Jia X, Xie Y. Integration of BOLD$\mathrm{fMRI}$ and DTI into radiation treatment planning for high-grade gliomas located near the primary motor cortexes and corticospinal tracts. Radiation oncology. 2015 Dec 1;10(1):64.

5. Makale MT, McDonald CR, Hattangadi-Gluth JA, Kesari S. Mechanisms of radiotherapy-associated cognitive disability in patients with brain tumours. Nature Reviews Neurology. 2017 Jan;13(1):52. 
6. Nelson MB, Compton P, Patel SK, Jacob E, Harper R. Central nervous system injury and neurobiobehavioral function in children with brain tumors: a review of the literature. Cancer nursing. 2013 Mar;36(2):E31.

7. Son Y, Yang M, Wang H, Moon C. Hippocampal dysfunctions caused by cranial irradiation: a review of the experimental evidence. Brain, behavior, and Immunity. 2015 Mar 1;45:287-96.

8. Liu R, Page M, Solheim K, Fox S, Chang SM (2009) Quality of life in adults with brain tumors: current knowledge and future directions. Neuro Oncol 11:330-339

9. McDuff SG, Taich ZJ, Lawson JD, Sanghvi P, Wong ET, Barker FG, Hochberg FH, Loeffler JS, Warnke PC, Murphy KT, Mundt AJ. Neurocognitive assessment following whole brain radiation therapy and radiosurgery for patients with cerebral metastases. Journal of Neurology, Neurosurgery \& Psychiatry. 2013 Dec 1;84(12):1384-91.

10. Bailey, C.C., Gnekow, A., Wellek, S., et al., 1995. Prospective randomized trial of chemotherapy given before radiotherapy in childhood medulloblastoma. International Society of Paediatric Oncology (SIOP) and the (German) Society of Paediatric Oncology (GPO): SIOP II. Med. Pediatr. Oncol. 25, $166-178$.

11. Padovani L, André N, Constine LS, Muracciole X. Neurocognitive function after radiotherapy for paediatric brain tumours. Nature Reviews Neurology. 2012 Oct;8(10):578-88.

12. Macedoni-Luksic, M., Jereb, B., Todorovski, L., 2003. Long-term sequelae in children treated for brain tumors: impairments, disability, and handicap. Pediatr. Hematol. Oncol. 20, 89- 101.

13. Ajithkumar T, Price S, Horan G, Burke A, Jefferies S. Prevention of radiotherapy-induced neurocognitive dysfunction in survivors of paediatric brain tumours: the potential role of modern imaging and radiotherapy techniques. The Lancet Oncology. 2017 Feb 1;18(2):e91-100.

14. Rutkowski S, Bode U, Deinlein F, et al. Treatment of early childhood medulloblastoma by postoperative chemotherapy alone. N Engl J Med 2005; 352: 978-86.

15. Pantelis E, Papadakis N, Verigos K, Stathochristopoulou I, Antypas C, Lekas L, Tzouras A, Georgiou E, Salvaras N. Integration of functional MRI and white matter tractography in stereotactic radiosurgery clinical practice. International Journal of Radiation Oncology* Biology* Physics. 2010 Sep 1;78(1):257-67.

16. Stancanello J, Cavedon C, Francescon P, et al BOLD fMRI integration into radiosurgery treatment planning of cerebral vascular malformations. Med Phys 2007;34:1176-1184.

17. Maruyama K, Kamada K, Shin M, et al Optic radiation tractography integrated into simulated treatment planning for Gamma Knife surgery. J Neurosurg 2007;107:721-726.

18. Maruyama K, Kamada K, Ata T, et al Tolerance of pyramidal tract to gamma knife radiosurgery based on diffusion-tensor tractography. Int J Radiat Oncol Biol Phys 2008;70:1330-1335.

19. Sun L, Qu B, Wang J, Ju Z, Zhang Z, Cui Z, Jack Y, Ling Z, Yu X, Pan L. Integration of functional MRI and white matter Tractography in CyberKnife radiosurgery. Technology in cancer research \& treatment. 2017 Dec;16(6):850-6. 
20. Ryan Fisicaro Nicole Petrovich Brennan and Andrei Holodny Chapter 29-Functional Magnetic Resonance Imaging In Handbook of Neuro-Oncology Neuroimaging (Second Edition) edited by Herbert B. Newton Academic Press San Diego 2016 Pages 317-325 ISBN 9780128009451.

21. Shevzov-Zebrun N, Brennan NM, Peck KK, Holodny Al. Advanced Functional Imaging: fMRI, PET, and MEG. InImage-Guided Neurosurgery 2015 Jan 1 (pp. 63-89). Academic Press.

22. Mahdavi A, Houshmand S , Oghabian MA, et al. Developing optimized fMRI protocol for clinical use: comparison of different language paradigms. J Magn Reson Imaging . $2011 ; 34: 413-419$.

\section{Figures}

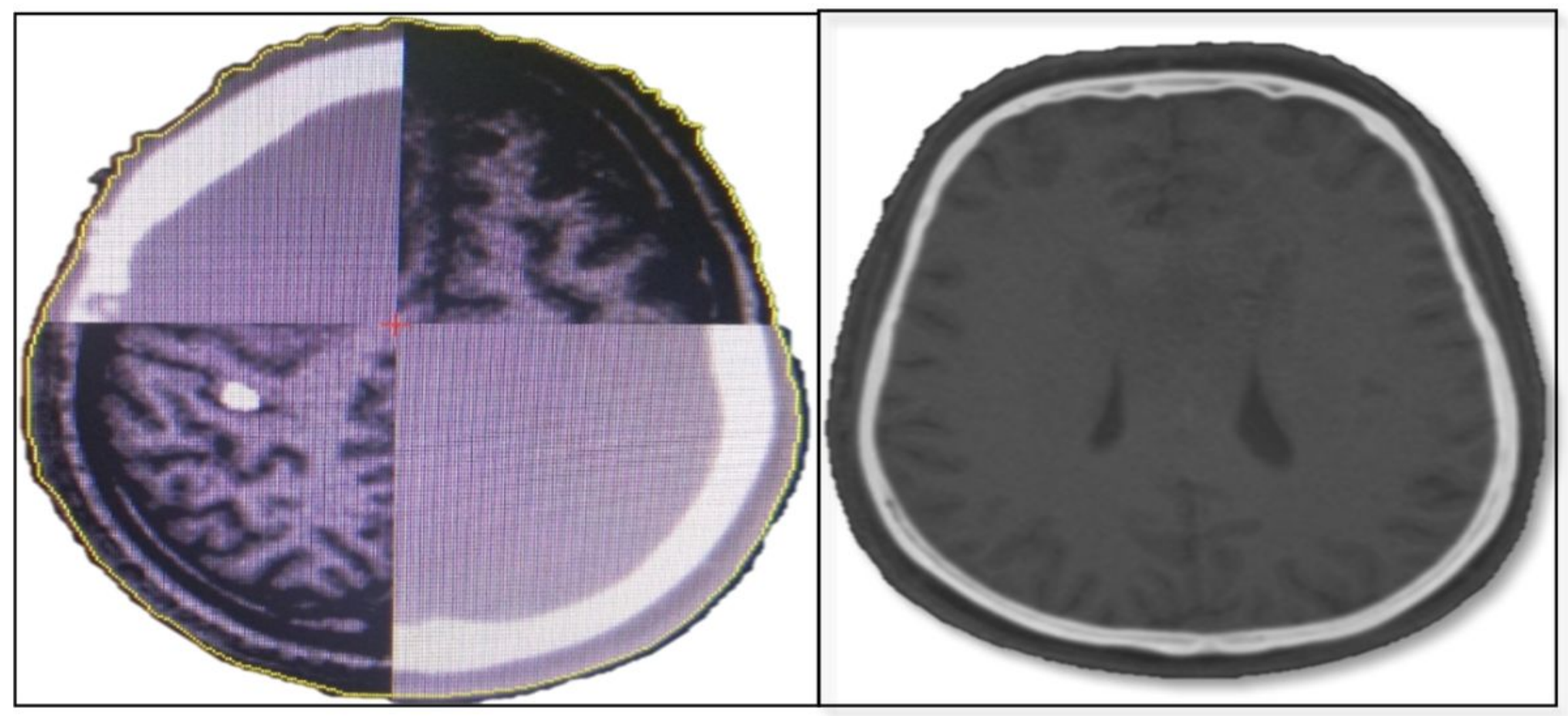

\section{Figure 1}

T1-weighted MR imaging and the corresponding axial CT after registration 


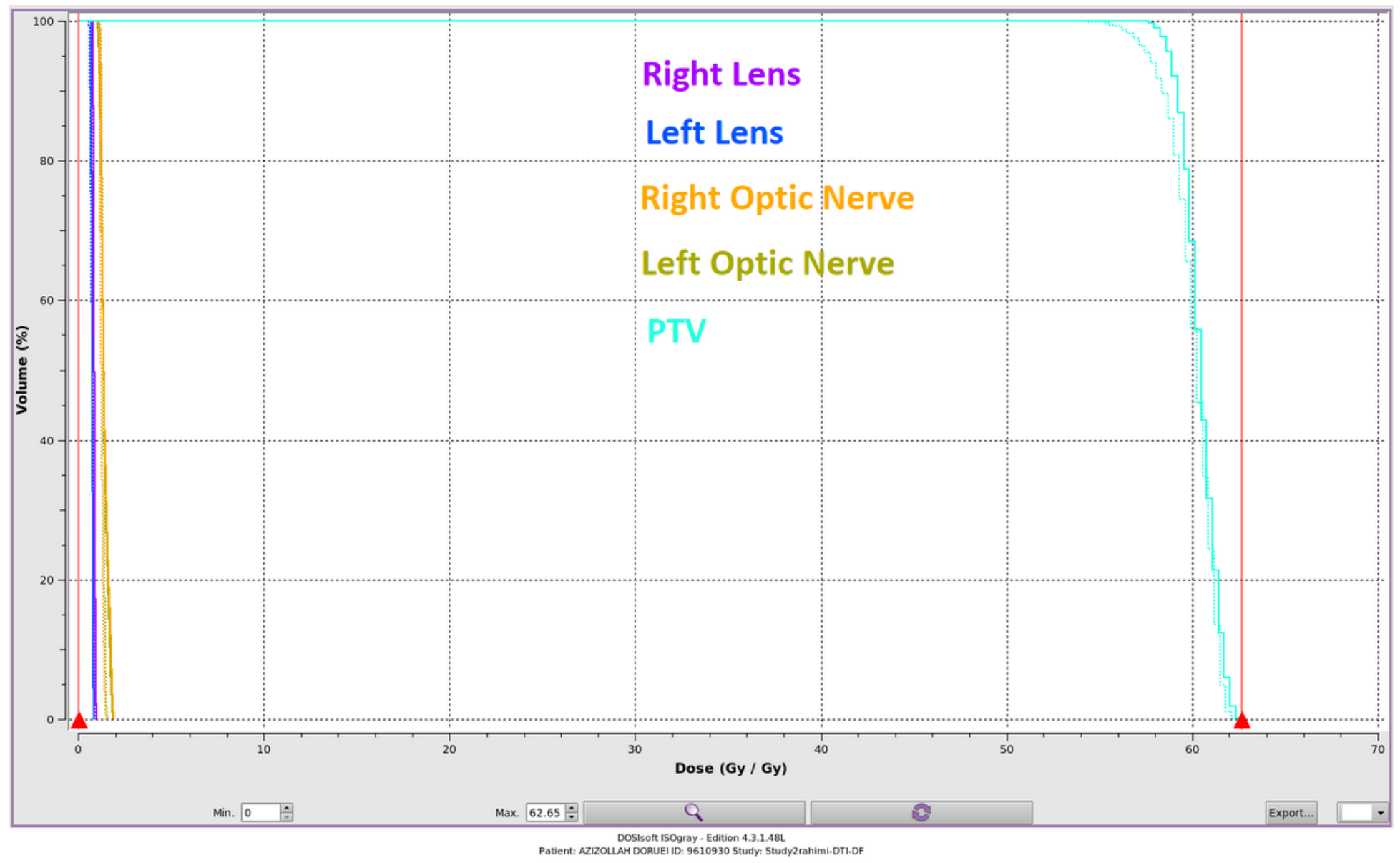

Figure 2

Dose-volume histograms for patient number 7 showing DVH curves for PTV, optic nerves, and lenses. (The solid line indicates the original plan and the Dashed line shows the optimized plan). 


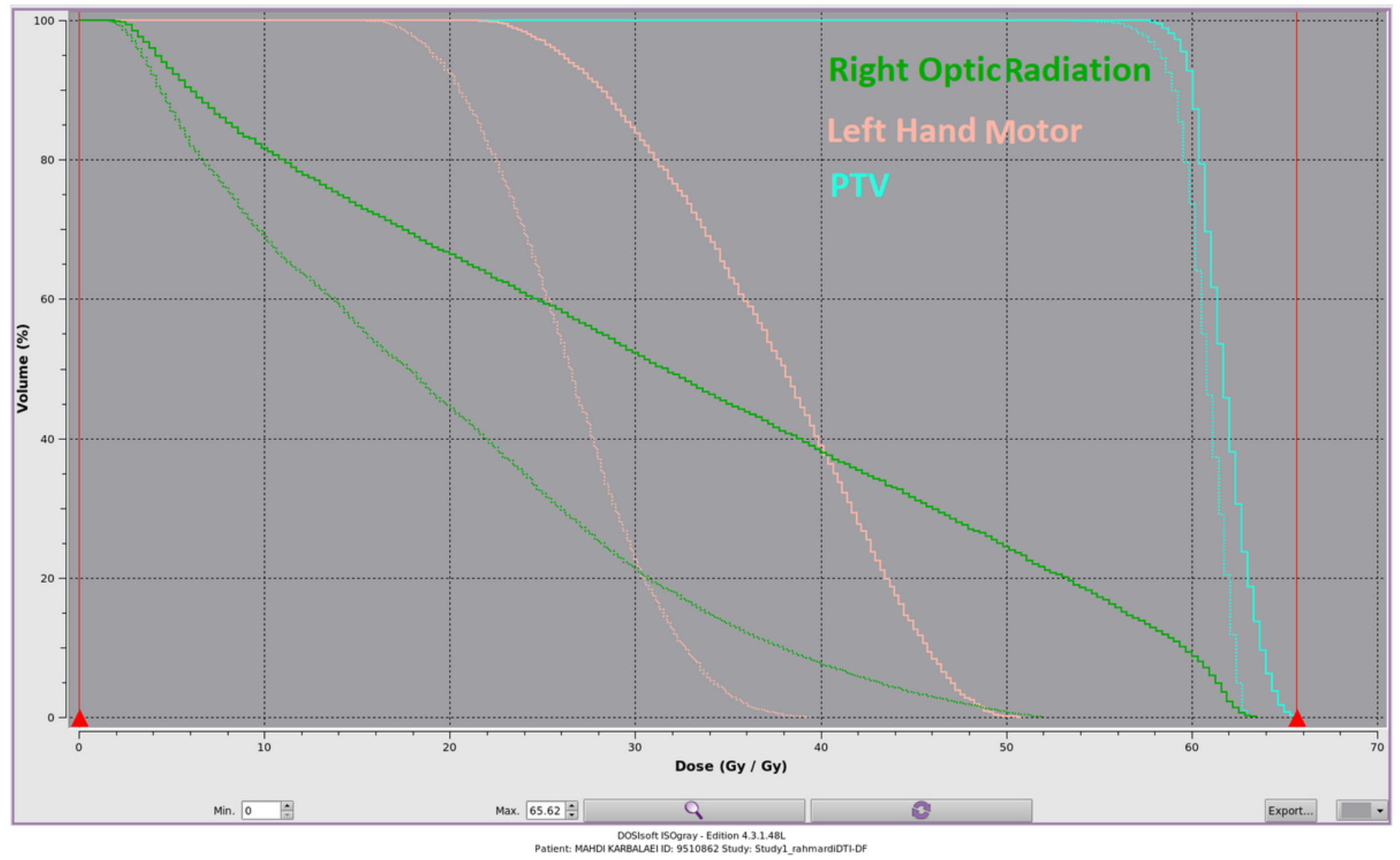

Figure 3

Dose-volume histograms for patient number 8 showing DVH curves to the PTV, right optic radiation, and left-hand motor. (The solid line indicates the original plan and the Dashed line shows the optimized plan) 